\title{
Ultrabright GeV Photon Source via Controlled Electromagnetic Cascades in Laser-Dipole Waves
}

\author{
A. Gonoskov, ${ }^{1,2,3}$ A. Bashinov, ${ }^{2}$ S. Bastrakov, ${ }^{3}$ E. Efimenko, ${ }^{2}$ A. Ilderton, ${ }^{1,4}$ \\ A. Kim, ${ }^{2}$ M. Marklund, ${ }^{1}$ I. Meyerov, ${ }^{3}$ A. Muraviev, ${ }^{2}$ and A. Sergeev ${ }^{2}$ \\ ${ }^{1}$ Department of Physics, Chalmers University of Technology, SE-41296 Gothenburg, Sweden \\ ${ }^{2}$ Institute of Applied Physics, Russian Academy of Sciences, Nizhny Novgorod 603950, Russia \\ ${ }^{3}$ Lobachevsky State University of Nizhni Novgorod, Nizhny Novgorod 603950, Russia \\ ${ }^{4}$ Centre for Mathematical Sciences, University of Plymouth, Plymouth PL4 8AA, United Kingdom \\ (Received 30 November 2016; revised manuscript received 23 June 2017; published 6 October 2017)
}

\begin{abstract}
Electromagnetic cascades have the potential to act as a high-energy photon source of unprecedented brightness. Such a source would offer new experimental possibilities in fundamental science, but in the cascade process radiation reaction and rapid electron-positron plasma production seemingly restrict the efficient production of photons to sub-GeV energies. Here, we show how to overcome these energetic restrictions and how to create a directed $\mathrm{GeV}$ photon source, with unique capabilities as compared to existing sources. Our new source concept is based on a controlled interplay between the cascade and anomalous radiative trapping. Using specially designed advanced numerical models supported with analytical estimates, we demonstrate that the concept becomes feasible at laser powers of around $7 \mathrm{PW}$, which is accessible at soon-to-be-available facilities. A higher peak power of $40 \mathrm{PW}$ can provide $10^{9}$ photons with $\mathrm{GeV}$ energies in a well-collimated 3-fs beam, achieving peak brilliance $9 \times 10^{24} \mathrm{ph} \mathrm{s}^{-1} \mathrm{mrad}^{-2} \mathrm{~mm}^{-2} / 0.1 \% \mathrm{BW}$.
\end{abstract}

DOI: 10.1103/PhysRevX.7.041003

Subject Areas: Optics, Plasma Physics

\section{INTRODUCTION}

One aim of upcoming high-intensity laser facilities $[1-5]$ is to provide new high-flux gamma-ray sources. Electromagnetic cascades [6-11] may serve for this, but are also known to limit both field strengths and particle energies [12], restricting efficient production of photons to sub-GeV energies [13-15]. Here, we show how to create a directed $\mathrm{GeV}$ photon source, enabled by a controlled interplay between the cascade and anomalous radiative trapping [16]. Using advanced 3D QED particle-in-cell (PIC) simulations [17] and analytic estimates, we show that the concept is feasible for planned [1,2] peak powers of 10-PW level.

Advances in high-intensity laser science offers opportunities for creating a new kind of high-flux gamma-ray source, based on the use of strong laser fields to accelerate particles and stimulate emission within a single optical cycle $[13-15,18,19]$. One of the most promising configurations is a standing wave formed by colliding laser pulses $[7,20,21]$. However, from a naive dynamical consideration one would expect particles to be expelled from the temporal and spatial regions which are optimal for energy gain

Published by the American Physical Society under the terms of the Creative Commons Attribution 4.0 International license. Further distribution of this work must maintain attribution to the author(s) and the published article's title, journal citation, and DOI. (i.e., the electric field antinodes). Furthermore, for intensities above $10^{24} \mathrm{~W} / \mathrm{cm}^{2}$ radiation losses prevent particles from reaching their potential maximum energy (during a single phase of acceleration). This limits the effective generation of photons to sub-GeV energies [12,22].

Our aim here is to find the optimal strategy for source creation using cascades triggered by several colliding laser pulses [7,20,21]. To do so we exploit the anomalous radiative trapping (ART) [16] of electrons and positrons in a dipole wave [23], the latter being the field configuration which provides the highest possible field strength for a given peak power of radiation. The dipole wave can be formed by orienting, timing, and focusing a number of laser pulses [16], as shown in Fig. 1. In this configuration, ART traps particles and makes them oscillate in spatiotemporal regions ideal for gaining a maximal possible energy. Furthermore, within each oscillation of the field the particles have, due to significant quantum effects, a high probability of emitting a large portion of their gained energy in the form of a single photon.

However, according to the analysis performed for singleparticle motion in a plane standing wave, triggering ART would require a total power of about $60 \mathrm{PW}$ [16]. Even if this were available, we see that already for lower powers a significant number of the generated high-energy photons can decay into $e^{+} e^{-}$pairs, giving rise to a cascade of pair production. As a result, before the peak intensity of the field is reached the cascade can generate an $e^{+} e^{-}$plasma dense enough to not only restrict the growth of the field 


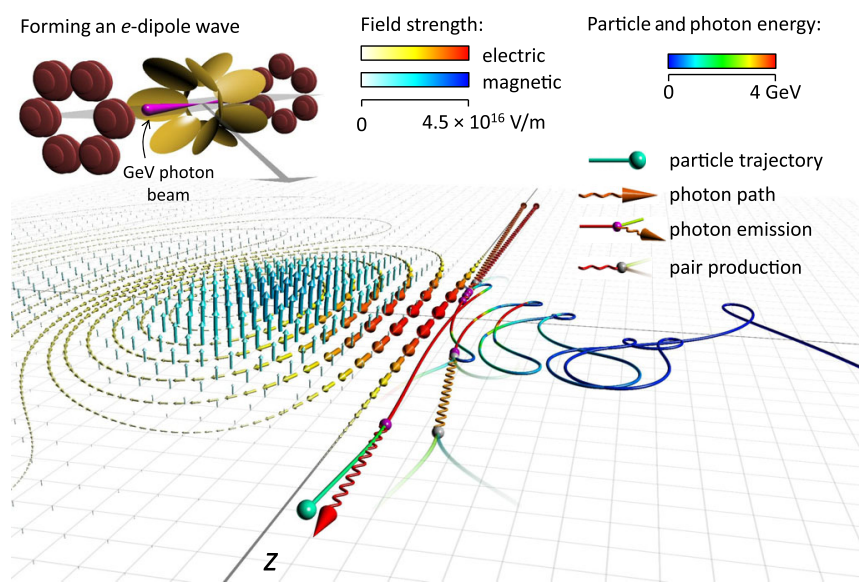

FIG. 1. Schematic illustration of the concept of anomalous radiative trapping and photon emission. In the top left we show how to generate a dipole wave using a set of off-axis parabolic mirrors (yellow) and 12 laser pulses (dark red). (See Ref. [16] for details and the reason for the choice of 12 mirrors.) The field structure of the dipole wave is shown in the left half of the horizontal plane (there is axial symmetry about the $z$ axis). We also show a trajectory of one of the seeding electrons taken from a simulation with $P=200 \mathrm{PW}$. Photons of energy above $3 \mathrm{GeV}$ are shown together with their emission events (magenta spheres). While some of these photons contribute to cascade development (gray spheres), others form a brilliant source emitted along the $z$ axis, also shown in the top left.

intensity [8] but also to terminate the favorable ART-regime dynamics. By assessing these processes with advanced simulations, we reveal here that for powers of the 10-PW level a new physical scenario arises in which the cascade and ART in fact induce and support each other. We demonstrate that controlling the cascade development by matching the laser pulse intensity and duration in realistic ranges makes it possible to create a unique and efficient photon source in which laser radiation is converted into a well-collimated flash of $\mathrm{GeV}$ photons with unprecedented brightness.

\section{DYNAMICS IN THE ART REGIME}

The key to our concept is matching the pulse duration, peak power, and initial particle density in the target, such that the maximal field intensity is reached just before collective plasma effects start to cause a significant reduction in the energy of generated photons. This requires that particle growth in the leading half of the laser pulse be restricted, such that the number of particles remains below a certain critical value $N_{\max }$, which would cause significant backreaction on the field. By ensuring that this constraint is fulfilled, we can arrange for a maximal number of particles to interact with the most intense part of the laser pulses, and emit a large number of high-energy photons, as desired. To perform a theoretical analysis and thus obtain estimates for the above constraint, we begin by describing how particle dynamics in the ART regime [16] governs the cascade.

The motion of particles in the ART regime, illustrated in Fig. 1, is quasiperiodic and consists of two qualitatively different phases. During the half-cycle when the electric field does not change sign, a trapped particle is accelerated and gains a kinetic energy of around $a m c^{2}$ before starting to emit frequently (hereafter, $a \approx 800 \sqrt{P}$ is the electric field amplitude in relativistic units for a dipole wave of total incoming power $P$ given hereafter in $\mathrm{PW}$ ); we refer to this as the acceleration phase. As the magnetic field strength rises, the particle loses a majority of its energy, having the possibility to emit photons with energies of up to $a m c^{2}$, and then starts gyrating; we refer to this as the turning phase, and this lasts until the electric field of the next acceleration phase starts to force the particle back in the opposite direction. Because of the probabilistic nature of emission in quantum electrodynamics, it may happen that the particles do not experience sufficient losses to be forced back, and hence leave the trapped state, as shown in Fig. 1.

Using estimates for the magnetic field strength in dipole waves [23], one can obtain that for powers of order $P=10$ $\mathrm{PW}$ the quantum efficiency parameter [24,25] $\chi$ attains values of order unity. This parameter is defined as the ratio $\chi=\gamma F_{\text {eff }} /\left(e E_{S}\right)$ of the force acting perpendicular to the direction of motion of the electron in its rest frame, to that force which it would experience in a field of SauterSchwinger strength, $E_{S}=m^{2} c^{3} / e \hbar \sim 10^{16} \mathrm{~V} / \mathrm{cm}(e$ and $\gamma$ are the electron charge and gamma factor, respectively). To calculate the quantum efficiency parameter for photons, one uses the energy in units of $m c^{2}$ instead of $\gamma$. The fact that $\chi \gtrsim 1$ means that a significant part of an electron's energy can be emitted as a single photon, which can then convert into an electron-positron $\left(e e^{+}\right)$pair. We estimate the threshold for when this leads to a pair production cascade as follows.

\section{INTERPLAY BETWEEN ART AND CASCADE}

A key result of this study is that pair production cascades and ART support each other, conspiring to lower the minimal total power required to trigger their mutual, self-sustained interaction. This effect has the following origin.

According to the analysis of ART performed in Ref. [16], for a single particle in a plane standing wave the dominance of radiation reaction leads to cophased oscillations along the electric field vector, accompanied by a net migration towards the electric field antinode. The mechanism of this migration starts to dominate over the attraction to the electric field nodes (normal radiative trapping) when the corresponding effective power exceeds $P_{\text {min }}^{s, p}=60 \mathrm{PW}$. Note that in a plane standing wave trapped particles can drift along the electric field vector while oscillating. However, in a dipole wave, the electromagnetic field, 
being strongly concentrated near the center, quickly drops along this direction. Thus, apart from the radial migration towards the center, the trapping requires confining the oscillations in the $z$ direction. However, the attraction point corresponds to the magnetic field node. Thus, in some vicinity of the $z$ axis the magnetic field is insufficiently strong to deplete the energy of outgoing particles after the acceleration phase, so they will inevitably escape. Moreover, this can happen everywhere due to the probabilistic nature of emission. However, the cascade onset can compensate for the leakage of particles from the trapped state. In this context, in a dipole wave and in the presence of cascades, the threshold for the trapping can be naturally defined as the power $P_{\text {min }}^{c, d}$ which leads to a self-sustained cascade; i.e., surpassing the threshold leads to the dominance of particle production in the trapped state over the leakage of particles from that state.

We now show how the cascade and ART support each other, leading to $P_{\min }^{c, d} \ll P_{\min }^{s, p}$. ART favors the cascade by bringing particles to the electric field antinode, where their energy gain during each oscillation is maximal. At the same time, the cascade produces new particles while others leave the trapped state. Moreover, this predominantly happens in the vicinity of the attraction point, further enhancing effective reallocation of particles towards this point. In such a way, for the multiparticle dynamics accompanied by a cascade, this effective trapping becomes sustained at a significantly lower level of power. This effective trapping is based on the following mechanism. If a particle leaving the trapped state produces photons that generate at least one pair on average, one of the produced particles is likely to be sent back into the trapped state. This is because on average the photons carry a quarter of the original particle's energy, while each of the produced particles carries on average one eighth. This is sufficiently low for the magnetic field to be able to deflect particles back into the trapped state, even if the field amplitude was insufficient to prevent the original particle from escaping. Hence, the cascade seeded by the original particle can provide another particle to replace it, and thus the process becomes self-sustaining. A lower power threshold $P_{\min }^{c, d} \ll P_{\min }^{s, p}$ is indeed observed in numerical studies; see Fig. 2(a). We now provide analytic estimates that demonstrate that the above mechanism can indeed be responsible for the threshold reduction.

In the considered configuration, particles and emitted photons propagate mostly almost along the $z$ axis (i.e., along the electric field vector). Thus, $\chi$ and consequently the rates of photon emission and pair production are predominantly determined by the magnetic field strength. The particles are then mostly produced around the instance when the magnetic field peaks, and in the spatial region where the particles and generated photons are in this instant of time; i.e., $z_{\mathrm{eff}} \approx \pm \lambda / 4, r_{\mathrm{eff}} \approx 0.15 \lambda$. [See Fig. $1(\mathrm{~b})$ in the Supplemental Material for details [26]. As one can see from Fig. 4 of Ref. [16], $r_{\text {eff }}$ does not depend strongly on $P$.]
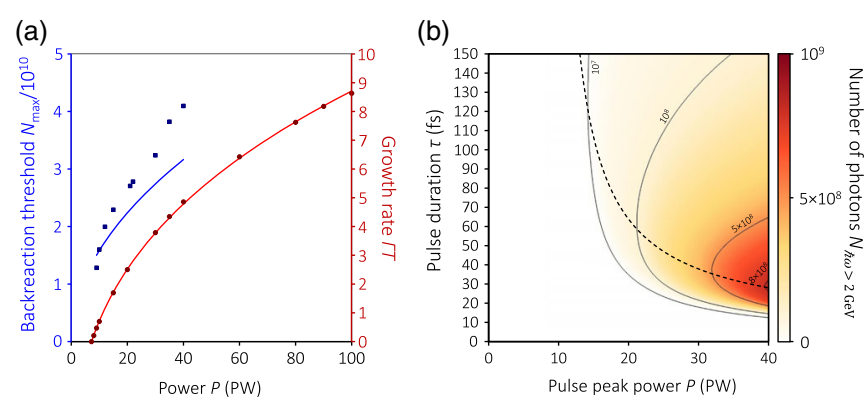

FIG. 2. Simulation results. (a) Rate of particle population growth (red, right-hand scale) and the dependency of the backreaction threshold (blue, left-hand scale), both as a function of power $P$. The analytical estimate for the backreaction threshold is shown with a solid blue line, and agrees very well with the numerical results shown as blue points. The accurate numerical approximation to the data points obtained from simulation is shown with a solid red curve, and this is used to derive the estimate Eq. (4). (b) The expected number of photons with energy above $2 \mathrm{GeV}$, as calculated based on Eq. (5) for a Gaussian pulse of total peak power $P$ and duration $\tau$. The analytically obtained optimal condition Eq. (4) is shown with the black dashed curve: this clearly runs through the center of the optimal region.

Using analytical expressions for the dipole wave profile [23], we can estimate that in this spatiotemporal region the effective magnetic field in relativistic units is $B_{\text {eff }} \approx 2.3 a r_{\text {eff }} / \lambda \approx 0.3 a$. For our estimate, we assume that a magnetic field of this strength acts for $1 / 8$ of the wave period $T$. We also assume that during the acceleration phase any particle produced during the previous turning phase gains a maximal possible energy $2 m c^{2} a$, without significant loses, and leaves the trapped state. However, while passing the turning phase the particle has $\chi \approx$ $0.6 a^{2}\left(\hbar \omega / m c^{2}\right) \approx P \gg 1$ and thus emits on average $n_{\mathrm{ph}} \approx$ $W_{\mathrm{ph}}^{\chi \gg 1} T / 8 \approx 2 P^{1 / 6}$ photons, where $W_{\mathrm{ph}}^{\chi \gg 1}=1.46 \alpha m^{2} c^{4} \chi^{2 / 3} /$ $\left(\hbar 2 a m c^{2}\right)$ is the emission rate in the limit of $\chi \gg 1$ and the dipole wave amplitude $a \approx 800 \sqrt{P}$. We assume that the particle maintains roughly the same energy and each of the emitted photons carries a quarter of this energy. As we will see below, this is reasonable because $n_{\mathrm{ph}}$ is of the order of unity for the values of $P$ around the threshold. Although the produced photons propagate having $\chi \approx P / 4 \sim 1$, we can estimate the average number of pairs produced by the photons using the small- $\chi$ approximation for the rate of pair production (which is known to be reasonably accurate also for $\chi \sim 1)$ :

$$
n_{\text {pair }} \approx 0.6 P^{2 / 3} \exp \left(-\frac{32}{3 P}\right)
$$

To obtain the threshold value for triggering the selfsustained process, we equate the average number of pairs to unity and obtain 


$$
P_{\text {min }}^{c, d} \approx 10 \mathrm{PW} .
$$

As we will see below [Fig. 2(a)], this analytically determined threshold value is in a good agreement with numerical results. This demonstrates consistency of our study and supports our interpretation of the process in terms of the underlying physics, as given above. However, the accuracy of this estimate is certainly insufficient for planning experiments. As we demonstrate in Supplemental Material, Sec. A [26], cascade evolution in our system is a highly complex process and a more accurate analysis is not amenable to simple analytical estimates. Thus, in the interest of providing accurate predictions, we turn to simulations.

\section{PULSE DURATION AND CASCADE CONTROL}

Pair production cascades are known to be highly complex processes [27-35] that require simulations for their analysis. In our study we use the 3D PIC code PICADOR [36,37] with quantum extensions [17] (see Supplemental Material, Sec. A [26]) to simulate cascade development in a dipole wave of constant incoming power $P$, for several values of $P$. We calculate the growth rate $\Gamma$ of particle number averaged over a half-period, using the relative increase of the number of trapped particles during a single turning phase. This takes into account both particle production and particles leaving the trapping state.

On time scales longer than a half-period, the particle number grows exponentially before backreaction sets in. For this exponential stage the rate $\Gamma$ depends only on $P$ and, according to the results shown in Fig. 2(a), may be approximately fitted to the curve $\Gamma(P) \approx 3.21 T^{-1}\left(P^{1 / 3}-P_{\min }^{1 / 3}\right)$, where $T$ is the laser period and $P_{\min } \simeq 7.2 \mathrm{PW}$ is the numerically determined threshold value required for the number of particles to grow. We integrate the pair production rate over the leading half of a laser pulse with Gaussian profile, FWHM duration $\tau$, and demand that by the instant that peak power is reached the number of trapped particles rises from $N_{0}=10$ (taken to guarantee seeding of the cascade) to $N_{\max }$, introduced above. By equating the Coulomb field of the trapped particles to $a / 2$, we estimate

$$
N_{\max } \approx 10^{9} \sqrt{P} .
$$

In Fig. 2(a), we demonstrate that this dependency (blue curve) is in reasonable agreement with the thresholds (blue points) determined in particular simulations as the instant when the growth rate starts to deviate from the initial value by more than $50 \%$. Using the estimate Eq. (3), we obtain the following optimal condition on the pulse duration $\tau$, at $P>P_{\min }$ :

$$
\frac{\tau}{T} \simeq \frac{2.23 \log \left[5 \times 10^{8} P^{1 / 2}\right]}{P^{0.59}-3.6} .
$$

This simple analytical expression may serve as guidance for the experimental implementation of the proposed concept.

In Fig. 2(b), we show the dependency Eq. (4) and demonstrate its agreement with the numerically calculated number of high-energy ( $\hbar \omega>2 \mathrm{GeV}$ ) photons expected for different values of $\tau$ and $P$. For this calculation we assume a slow variation of the wave amplitude within the pulse and describe the discreet evolution of the number of trapped particles $N_{e e^{+}}$and the number of generated photons $N_{\text {ph }}$ (which escape from the strong-field region having energy $\hbar \omega>2 \mathrm{GeV}$ ) at the instances of $E=0$ using the following equations:

$$
\begin{aligned}
N_{e e^{+}}(t+T / 2) & =N_{e e^{+}}(t) Y\left(P(t+T / 4), N_{e e^{+}}(t)\right), \\
N_{\mathrm{ph}}(t+T / 2) & =N_{\mathrm{ph}}(t)+N_{e e^{+}}(t) X\left(P(t+T / 4), N_{e e^{+}}(t)\right),
\end{aligned}
$$

where the functions $X\left(P, N_{e e^{+}}\right)$and $Y\left(P, N_{e e^{+}}\right)$are defined as the relative increase in the number of particles and the number of generated photons within a single half-period between instances of $E=0$, for the current value of power $P$ and number of particles $N_{e e^{+}}$at the beginning of the interval. To determine $X\left(P, N_{e e^{+}}\right)$and $Y\left(P, N_{e e^{+}}\right)$we use the data obtained in the set of numerical experiments with constant power $P$ and interpolate the values in other points (see Fig. 3). Next, we calculate the expected number of photons using Eq. (5).

As can be seen from Fig. 2(b), a large number of energetic photons are produced and escape the fields, despite a rapid cascade process. It is worth explaining the reason for this in some detail, as it is key to the unique properties of our source. There are three factors favoring the escape of the photons. First, within the acceleration phase particles move mostly linearly without too strong acceleration across their motion. This reduces the overall rate of radiation losses prior to reaching high energies. Second, due to high values of $\chi$ the interval between
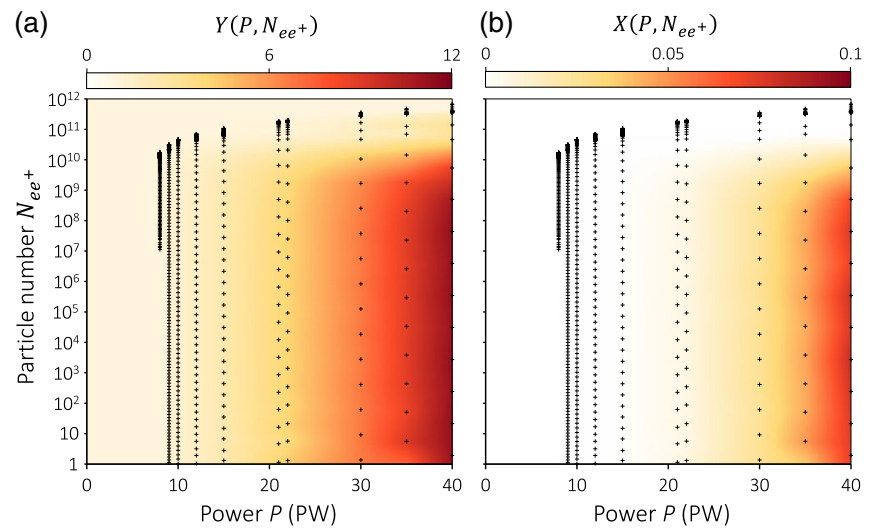

FIG. 3. The functions (a) $X\left(P, N_{e e^{+}}\right)$and (b) $Y\left(P, N_{e e^{+}}\right)$ interpolated from the values calculated from the data of simulations with constant power $P$ at the instances of $E=0$ (crosses). 


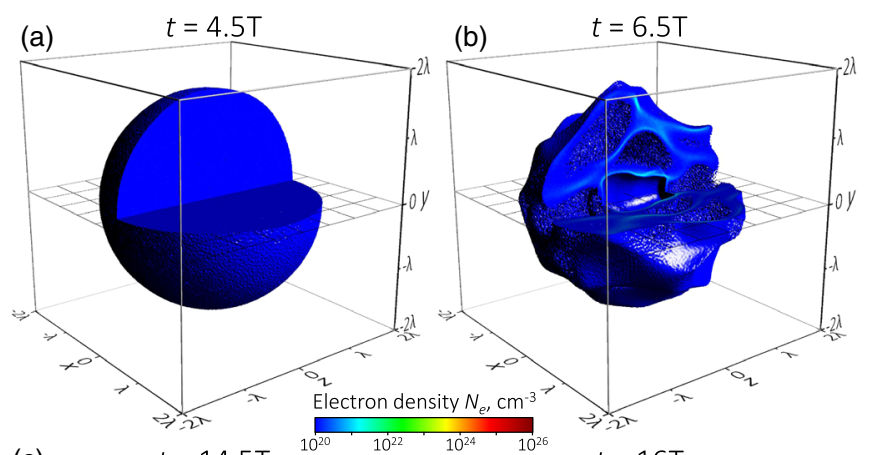

(c)

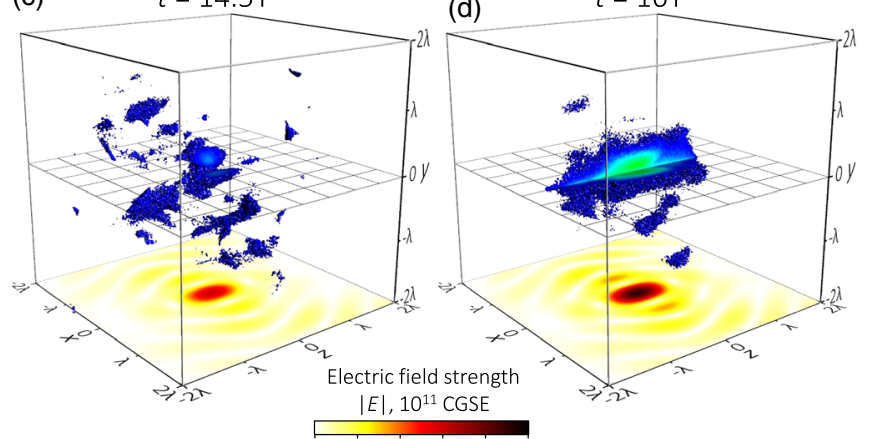

(e) $\quad t=17.5 \mathrm{~T} \quad$\begin{tabular}{llllll}
\hline & 1 & 2 & 3 & 4 & 5
\end{tabular}

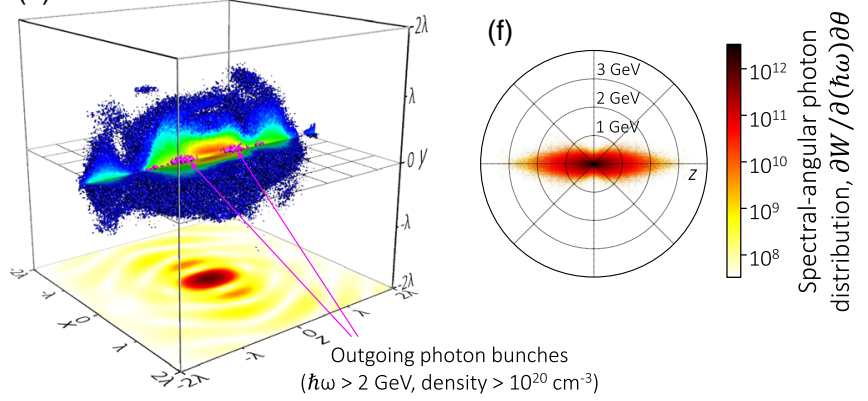

(g)

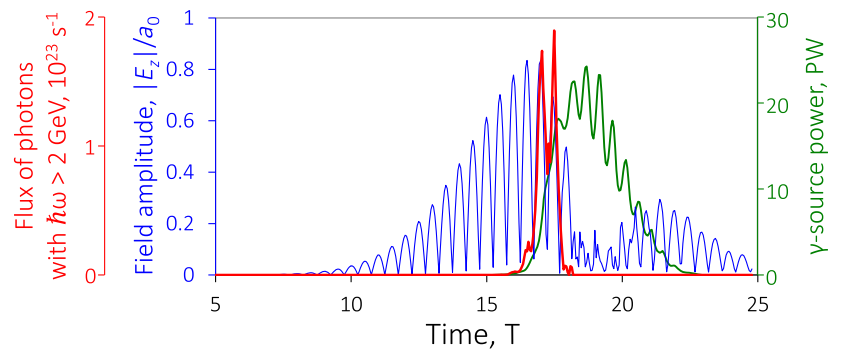

FIG. 4. The results of 3D PIC simulations for a dipole wave formed by 12 slightly dephased laser pulses of 15 -fs duration (FWHM for intensity) and total peak power of $40 \mathrm{PW}$. The electron density $>10^{20} \mathrm{~cm}^{-3}$ and electric field strength in the plane $y=0$ (shifted downward) are shown for several instances: (a) the initial target, (b) the target affected by the leading edge of the pulses, (c) ART beginning to trap particles and start the cascade, (d) the cascade development, and (e) the generation of $\mathrm{GeV}$ photons. The overall angular-resolved spectrum of emitted photons is shown in (f). Panel (g) shows the temporal evolution of the field strength in the center (blue line), the total power of generated photons (green line), and the flux of photons with energy $>2 \mathrm{GeV}$ (red line).
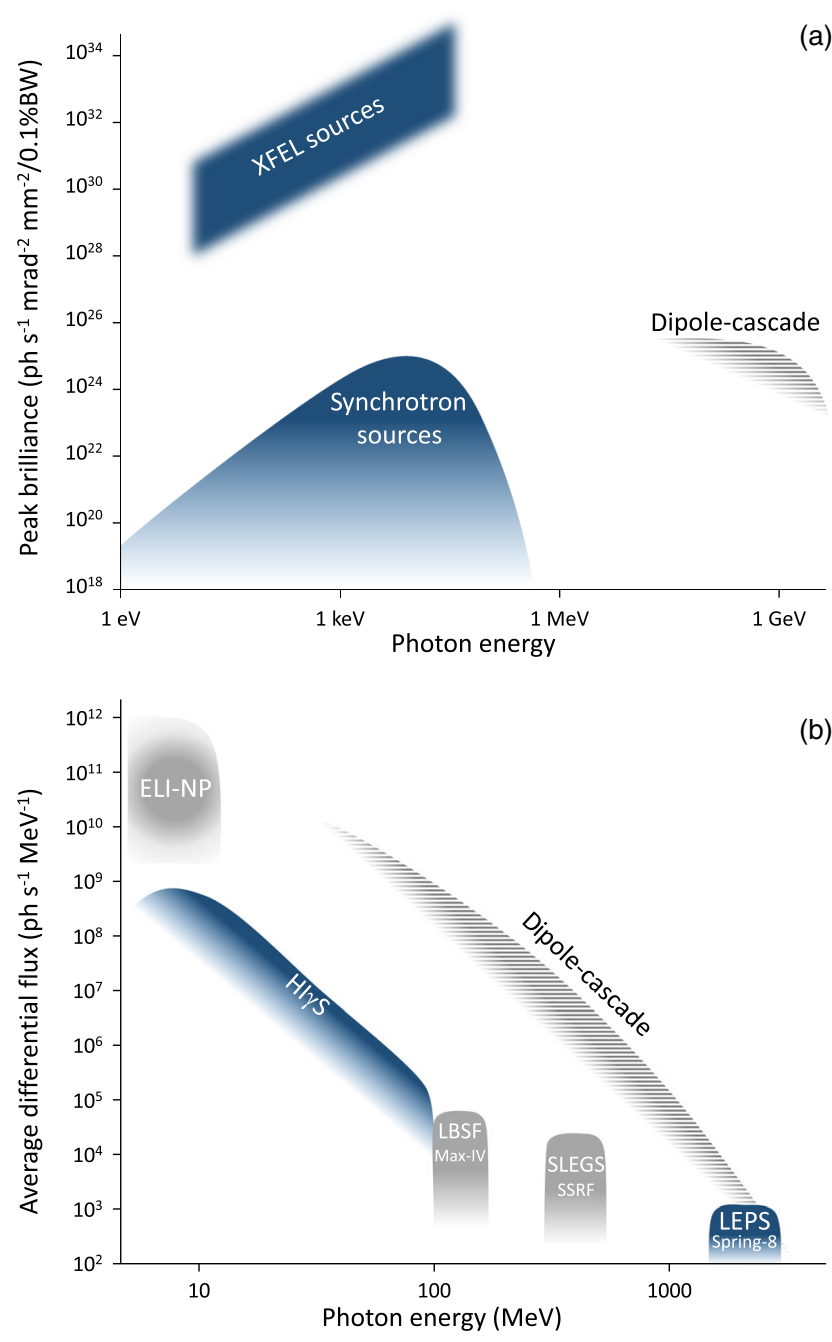

FIG. 5. Comparison of the proposed source with existing sources, in terms of (a) peak brilliance and (b) average flux. The plots represent schematically summarized properties of existing (blue) as well as upcoming or planned (solid gray) facilities. The gray hatched areas show the prospects of our proposed source, estimated based on the results of 3D PIC simulations.

emissions can be relatively long, enabling a reasonable probability for the particle motion without any radiation losses (especially in the vicinity of the $z$ axis; see Fig. 5), which is also known as quantum quenching of radiation reaction [38]. Thus, a particle has a reasonable chance to gain high energy and emit its significant part in the form of a single energetic photon instead of being constantly depleted by the emission of low-energy photons. Third, the emitted energetic photons have a significant probability to escape without decaying into pairs. We can estimate this probability using the large- $\chi$ approximation for the rate of pair production and the assumptions used for obtaining the estimate Eq. (2): 


$$
P_{\text {escape }} \approx \exp \left[-0.65\left(\frac{\epsilon_{\mathrm{ph}}}{a m c^{2}}\right)^{-1 / 3} P^{1 / 6}\right]
$$

For $P=40 \mathrm{PW}$ and the photon energy $\epsilon_{\mathrm{ph}}=a m c^{2} \approx$ $2.5 \mathrm{GeV}$, we have $P_{\text {escape }} \approx 30 \%$. Note that, according to the obtained expression, photons of higher energy have a higher chance to escape than those with low energy, which thus more efficiently sustain the cascade. In such a way, the considered configuration is favorable for converting laser energy into high-energy photons.

\section{SOURCE PROPERTIES}

Having now identified the required pulse parameters, we provide a concrete example of the possible source properties using a comprehensive PIC simulation. (For full simulation details, see Supplemental Material, Sec. B [26].) The results are shown in Fig. 4 (see also the video in the Supplemental Material [26]). We form the dipole wave using 12 laser pulses with a total peak power of $40 \mathrm{PW}$. (Using 12 beams efficiently mimics the ideal dipole wave; see Ref. [16]. With the advent of more powerful systems, beams could be split into the needed number to mimic the dipole wave.) Each laser pulse has 15-fs duration (FWHM for intensity), which is matched to the initial particle density of the target (a uniform sphere with diameter $3 \mu \mathrm{m}$ and density $10^{20} \mathrm{~cm}^{-3}$ ). Instead of the considered target, in reality adjusting the density of stray particles in the chamber can be used to trigger the cascade starting from an arbitrarily small number of particles. (This is because the cascade can even be prevented by choosing high enough vacuum [10,39].) To account for realistic experimental conditions, and so demonstrate the robustness of our concept against realistic imperfections of focusing geometry, the pulses are randomly dephased within $1 / 30$ of the wave period. The simulation shows that the concept provides $10^{9}$ photons with energies $\hbar \omega>2 \mathrm{GeV}$ in a well-collimated beam $\left(<5^{\circ}\right)$ and of just 3 -fs duration, achieving peak flux of $1.9 \times 10^{23} \mathrm{ph} \mathrm{s}^{-1}$, brightness $7.4 \times 10^{26} \mathrm{ph} \mathrm{s}^{-1} \mathrm{mrad}^{-2} \mathrm{~mm}^{-2}$, and brilliance $9 \times 10^{24} \mathrm{ph} \mathrm{s}^{-1} \mathrm{mrad}^{-2} \mathrm{~mm}^{-2} / 0.1 \% \mathrm{BW}$.

We can now compare the capabilities of the proposed source with those of existing sources. As one can see from Fig. 5(a), the proposed source has the unique capability of achieving high peak brilliance in an energy range unachievable for conventional sources. In fact, the $\mathrm{GeV}$ range of photon energies is currently achievable only with sources based on the inverse Compton scattering of laser photons from energetic particles produced by large-scale accelerators. These sources provide the possibility of accumulating statistics over a large number of collisions; thus, instead of brilliance they are characterized by average flux. Assuming an interval of $1000 \mathrm{~s}$ between laser shots, we provide a comparison in terms of average flux in Fig. 5(b). One can see that the proposed source has a wide energy coverage and can exceed the average flux of existing sources by several orders of magnitude [40]. Moreover, the considered conventional characteristics are related to particular types of sources or applications and do not reflect what is perhaps the most interesting aspect of the source, namely, that the produced GeV photons occupy an extremely small volume of space $\left(\sim 10^{-2} \mu \mathrm{m}\right.$ for each burst), reaching a peak density of the order of $10^{21} \mathrm{~cm}^{-3}$ [see Fig. 4(e)]. Such a source, with extraordinary high flux of $\mathrm{GeV}$ photons with a broad spectrum, would be a powerful tool for studying fundamental electromagnetic [25] processes, and will open qualitatively new possibilities for studying photonuclear processes $[1,41,42]$, such as triggering multiple transitions between short-lived states. One could also take advantage of the even higher photon flux in the focal region, by placing nuclear targets in or as close as possible to the focal spot.

TABLE I. Validation of the concept's feasibility at upcoming facilities. Here, we show the results of 3D PIC simulations for low values of the total peak power (of all beams forming the dipole wave). The number of photons produced is calculated for different energy ranges. For these ranges we also express the total radiated energy as a percentage of the initial total energy of the laser radiation.

\begin{tabular}{|c|c|c|c|c|c|}
\hline \multirow{2}{*}{$\begin{array}{l}\text { Total } \\
\text { power }(\mathrm{PW})\end{array}$} & \multirow{2}{*}{$\begin{array}{c}\text { Pulse } \\
\text { duration (fs) }\end{array}$} & \multirow{2}{*}{$\begin{array}{c}\text { Target } \\
\text { density, }\left(\mathrm{cm}^{-3}\right)\end{array}$} & \multirow[b]{2}{*}{ Focusing model (deviations) } & \multicolumn{2}{|c|}{ Number of photons (efficiency) } \\
\hline & & & & All & $>1 \mathrm{GeV}$ \\
\hline 8 & \multirow{10}{*}{30} & $2 \times 10^{22}$ & Ideal & $4.3 \times 10^{12}(19 \%)$ & $1.1 \times 10^{9}(0.07 \%)$ \\
\hline \multirow{4}{*}{10} & & $10^{22}$ & & $1.2 \times 10^{12}(4.2 \%)$ & $6.7 \times 10^{8}\left(3.5 \times 10^{-4}\right)$ \\
\hline & & $10^{23}$ & 12 beams (space and phase) & $1.5 \times 10^{13}(42 \%)$ & $4.7 \times 10^{7}\left(2.3 \times 10^{-5}\right)$ \\
\hline & & $10^{16}$ & & $8.8 \times 10^{5}\left(2.7 \times 10^{-8}\right)$ & $1.8 \times 10^{3}\left(6.6 \times 10^{-10}\right)$ \\
\hline & & $10^{22}$ & Ideal & $1.5 \times 10^{13}(42 \%)$ & $1.4 \times 10^{10}(0.49 \%)$ \\
\hline \multirow{4}{*}{15} & & $10^{22}$ & & $8.2 \times 10^{12}(23 \%)$ & $7.8 \times 10^{9}(0.28 \%)$ \\
\hline & & $2 \times 10^{22}$ & 12 beams (space and phase) & $1.3 \times 10^{13}(35 \%)$ & $6.7 \times 10^{9}(0.16 \%)$ \\
\hline & & $3 \times 10^{22}$ & & $1.6 \times 10^{13}(41 \%)$ & $4.6 \times 10^{9}(0.16 \%)$ \\
\hline & & $10^{22}$ & 12 beams (phase) & $1.8 \times 10^{13}(37 \%)$ & $1.5 \times 10^{10}(0.39 \%)$ \\
\hline 20 & & $10^{22}$ & 12 beams (space and phase) & $1.7 \times 10^{13}(33 \%)$ & $1.2 \times 10^{10}(0.33 \%)$ \\
\hline
\end{tabular}


TABLE II. Validation of the concept's robustness. Here, we show the results of 3D PIC simulations with and without random deviations in phase and focus location for the 12 beams forming the dipole wave. The results are also compared with those for an ideal dipole wave. The results are presented as in Table I.

\begin{tabular}{|c|c|c|c|c|c|c|}
\hline \multirow{2}{*}{$\begin{array}{l}\text { Total } \\
\text { power } \\
(\mathrm{PW})\end{array}$} & \multirow{2}{*}{$\begin{array}{c}\text { Pulse } \\
\text { duration } \\
\text { (fs) }\end{array}$} & \multirow{2}{*}{$\begin{array}{c}\text { Target } \\
\text { density, } \\
\left(\mathrm{cm}^{-3}\right)\end{array}$} & \multirow[b]{2}{*}{ Focusing model (deviations) } & \multicolumn{3}{|c|}{ Number of photons (efficiency) } \\
\hline & & & & All & $>100 \mathrm{MeV}$ & $>2 \mathrm{GeV}$ \\
\hline \multirow{7}{*}{40} & 15 & $10^{13}$ & Ideal & $8.1 \times 10^{11}(1.2 \%)$ & $1.4 \times 10^{11}(0.9 \%)$ & $2.5 \times 10^{7}\left(1.4 \times 10^{-5}\right)$ \\
\hline & & $10^{19}$ & Ideal & $4 \times 10^{13}(34 \%)$ & $3.7 \times 10^{12}(18 \%)$ & $7.6 \times 10^{8}\left(4.4 \times 10^{-4}\right)$ \\
\hline & & $10^{13}$ & 12 beams (space and phase) & $2.3 \times 10^{13}(21 \%)$ & $2.1 \times 10^{12}(12 \%)$ & $4.7 \times 10^{8}\left(2.6 \times 10^{-4}\right)$ \\
\hline & & $10^{20}$ & 12 beams (phase) & $3.8 \times 10^{13}(30 \%)$ & $3 \times 10^{12}(15 \%)$ & $3.7 \times 10^{8}\left(2.1 \times 10^{-4}\right)$ \\
\hline & & $10^{2}$ & 12 beams (space and phase) & $3.3 \times 10^{13}(28 \%)$ & $2.8 \times 10^{12}(15 \%)$ & $3.8 \times 10^{8}\left(2.1 \times 10^{-4}\right)$ \\
\hline & 25 & $10^{20}$ & 12 beams (space and phase) & $8.7 \times 10^{13}(39 \%)$ & $6.3 \times 10^{12}(18 \%)$ & $2.9 \times 10^{8}\left(9.7 \times 10^{-5}\right)$ \\
\hline & 25 & $10^{21}$ & 12 beams (space and phase) & $10^{14}(46 \%)$ & $7.5 \times 10^{12}(22 \%)$ & $1.3 \times 10^{8}\left(4.4 \times 10^{-5}\right)$ \\
\hline
\end{tabular}

\section{A. Experimental prospects}

We perform a series of simulations to investigate the robustness of the proposed concept and the prospects of performing a proof-of-principle experiment at soon-to-beavailable facilities [1,2]. For these purposes, we consider a 12-beam model mimicking an ideal dipole wave, and further introduce random deviations within $\pi / 15$ for the phase and $\lambda / 30$ for the position of the focus of each beam. Additionally, we vary the total peak power of all laser channels and the initial target density (i.e., the number of particles delivered in some way to the focal region in order to seed the cascade). The results are presented in Tables I and II. For each case, the interplay between the cascade and ART is triggered. In real experiments it will be possible to confirm this by detecting a well-collimated emission of high-energy photons or energetic electrons or positrons along the symmetry axis. These results demonstrate that the proposed concept is sufficiently robust and feasible to be verified in proof-of-principle experiments at those upcoming facilities that aim for a total peak power of $10 \mathrm{PW}$.

\section{ACKNOWLEDGMENTS}

The authors acknowledge support from the Russian Science Foundation Project No. 16-12-10486 (A. G., A. B., E.E., A. M., A. K.), the Russian Foundation for Basic Research Project No. 15-37-21015 (developments for the QED-PIC simulations, A. G., A. M, S. B., I. M.), the EU Horizon 2020 programme under the Marie Skł odowskaCurie Project No. 701676 (A. I.), the Olle-Engkvist Foundation, Grant No. 2014/744 (A. I.), and the Knut and Alice Wallenberg Foundation (A. G., A. I., M. M.). The simulations were performed on resources provided by the Swedish National Infrastructure for Computing (SNIC) and the Joint Supercomputer Center of RAS.

A. G. and A. B. contributed equally to this work.

[1] ELI-NP, www.eli-np.ro.

[2] ELI beamlines, www.eli-beams.eu.
[3] XCELS, www.xcels.iapras.ru.

[4] VULCAN, www.clf.stfc.ac.uk/Pages/The-Vulcan-10Petawatt-Project.aspx.

[5] ELI, www.extreme-light-infrastructure.eu/; ELI delivery consortium, https://eli-laser.eu/scienceapplications/ secondary-sources/.

[6] A. R. Bell and J.G. Kirk, Possibility of Prolific Pair Production with High-Power Lasers, Phys. Rev. Lett. 101, 200403 (2008).

[7] S. S. Bulanov, V. D. Mur, N. B. Narozhny, J. Nees, and V. S. Popov, Multiple Colliding Electromagnetic Pulses: A Way to Lower the Threshold of $e^{+} e^{-}$Pair Production from Vacuum, Phys. Rev. Lett. 104, 220404 (2010).

[8] A. M. Fedotov, N. B. Narozhny, G. Mourou, and G. Korn, Limitations on the Attainable Intensity of High Power Lasers, Phys. Rev. Lett. 105, 080402 (2010).

[9] E. N. Nerush, I. Yu. Kostyukov, A. M. Fedotov, N. B. Narozhny, N. V. Elkina, and H. Ruhl, Laser Field Absorption in Self-Generated Electron-Positron Pair Plasma, Phys. Rev. Lett. 106, 035001 (2011).

[10] M. Tamburini, A. Di Piazza, and C. H. Keitel, Laser-PulseShape Control of Seeded QED Cascades, Sci. Rep. 7, 5694 (2017).

[11] T. Grismayer, M. Vranic, J. L. Martins, R. Fonseca, and L. O. Silva, Seeded QED Cascades in Counterpropagating Laser Pulses, Phys. Rev. E 95, 023210 (2017).

[12] S. V. Bulanov, T. Esirkepov, J. Koga, and T. Tajima, Interaction of Electromagnetic Waves with Plasma in the Radiation-Dominated Regime, Plasma Phys. Rep. 30, 196 (2004).

[13] C. P. Ridgers, C. S. Brady, R. Duclous, J. G. Kirk, K. Bennett, T.D. Arber, and A. R. Bell, Dense ElectronPositron Plasmas and Bursts of Gamma-Rays from Laser-Generated Quantum Electrodynamic Plasmas, Phys. Plasmas 20, 056701 (2013).

[14] M. Jirka, O. Klimo, S. V. Bulanov, T.Zh. Esirkepov, E. Gelfer, S. S. Bulanov, S. Weber, and G. Korn, Electron Dynamics and $\gamma$ and $e^{+} e^{-}$Production by Colliding Laser Pulses, Phys. Rev. E 93, 023207 (2016).

[15] T. Grismayer, M. Vranic, J. L. Martins, R. A. Fonseca, and L. O. Silva, Laser Absorption via Quantum Electrodynamics Cascades in Counter propagating Laser Pulses, Phys. Plasmas 23, 056706 (2016). 
[16] A. Gonoskov, A. Bashinov, I. Gonoskov, C. Harvey, A. Ilderton, A. Kim, M. Marklund, G. Mourou, and A. Sergeev, Anomalous Radiative Trapping in Laser Fields of Extreme Intensity, Phys. Rev. Lett. 113, 014801 (2014).

[17] A. Gonoskov, S. Bastrakov, E. Efimenko, A. Ilderton, M. Marklund, I. Meyerov, A. Muraviev, A. Sergeev, I. Surmin, and E. Wallin, Extended Particle-in-Cell Schemes for Physics in Ultrastrong Laser Fields: Review and Developments, Phys. Rev. E 92, 023305 (2015).

[18] M. Vranic, T. Grismayer, R. A. Fonseca, and L. O. Silva, Electron-Positron Cascades in Multiple-Laser Optical Traps, Plasma Phys. Controlled Fusion 59, 014040 (2017).

[19] Z. Gong, R. H. Hu, Y. R. Shou, B. Qiao, C. E. Chen, X. T. He, S. S. Bulanov, T. Zh. Esirkepov, S. V. Bulanov, and X. Q. Yan, High Efficiency Gamma-Ray Flash Generation via Multiple Compton Scattering, Phys. Rev. E 95, 013210 (2017).

[20] S. S. Bulanov, T. Zh. Esirkepov, A. G. R. Thomas, J. K. Koga, and S. V. Bulanov, Schwinger Limit Attainability with Extreme Power Lasers, Phys. Rev. Lett. 105, 220407 (2010).

[21] E. G. Gelfer, A. A. Mironov, A. M. Fedotov, V. F. Bashmakov, E. N. Nerush, I. Yu. Kostyukov, and N. B. Narozhny, Optimized Multibeam Configuration for Observation of QED Cascades, Phys. Rev. A 92, 022113 (2015).

[22] C. Yu et al., Ultrahigh Brilliance Quasi-Monochromatic $\mathrm{MeV} \gamma$-Rays Based on Self-Synchronized All-Optical Compton Scattering, Sci. Rep. 6, 29518 (2016).

[23] I. Gonoskov, A. Aiello, S. Heugel, and G. Leuchs, Dipole Pulse Theory: Maximizing the Field Amplitude from 4 Focused Laser Pulses, Phys. Rev. A 86, 053836 (2012).

[24] V. Ritus, Quantum Effects of the Interaction of Elementary Particles with an Intense Electromagnetic Field, J. Sov. Laser Res. 6, 497 (1985).

[25] A. Di Piazza, C. Muller, K. Z. Hatsagortsyan, and C. H. Keitel, Extremely High-Intensity Laser Interactions with Fundamental Quantum Systems, Rev. Mod. Phys. 84, 1177 (2012).

[26] See Supplemental Material at http://link.aps.org/ supplemental/10.1103/PhysRevX.7.041003 for more details on the cascade specificity (Sec. A), numerical model (Sec. B) and analytical calculations (Sec. C).

[27] E. N. Nerush, V. F. Bashmakov, and I. Yu. Kostyukov, Analytical Model for Electromagnetic Cascades in Rotating Electric Field, Phys. Plasmas 18, 083107 (2011).

[28] N. V. Elkina, A. M. Fedotov, I. Yu. Kostyukov, M. V. Legkov, N. B. Narozhny, E. N. Nerush, and H. Ruhl, QED Cascades Induced by Circularly Polarized Laser Fields, Phys. Rev. ST Accel. Beams 14, 054401 (2011).
[29] V. F. Bashmakov, E. N. Nerush, I. Yu. Kostyukov, A. M. Fedotov, and N. B. Narozhny, Effect of Laser Polarization on Quantum Electrodynamical Cascading, Phys. Plasmas 21, 013105 (2014).

[30] A. A. Mironov, N. B. Narozhny, and A. M. Fedotov, Collapse and Revival of Electromagnetic Cascades in Focused Intense Laser Pulses, Phys. Lett. A 378, 3254 (2014).

[31] T. Zh. Esirkepov, S. S. Bulanov, J. K. Koga, M. Kando, K. Kondo, N. N. Rosanov, G. Korn, and S. V. Bulanov, Attractors and Chaos of Electron Dynamics in Electromagnetic Standing Waves, Phys. Lett. A 379, 2044 (2015).

[32] J. G. Kirk, Radiative Trapping in Intense Laser Beams, Plasma Phys. Controlled Fusion 58, 085005 (2016).

[33] A. V. Bashinov, P. Kumar, and A. V. Kim, Particle Fynam$i$ cs and Spatial $e^{-}-e^{+}$Density Structures at QED Cascading in Circularly Polarized Standing Waves, Phys. Rev. A 95, 042127 (2017).

[34] A. V. Bashinov, A. V. Kim, and A. M. Sergeev, Impact of Quantum Effects on Relativistic Electron Motion in a Chaotic Regime, Phys. Rev. E 92, 043105 (2015).

[35] S. V. Bulanov, T. Zh. Esirkepov, J. K. Koga, S. S. Bulanov, Z. Gong, X. Q. Yan, and M. Kando, Charged Particle Dynamics in Multiple Colliding Electromagnetic Waves. Survey of Random Walk, Lévy Flights, Limit Circles, Attractors and Structurally Determinate Patterns, J. Plasma Phys. 83, 905830202 (2017).

[36] S. Bastrakov, R. Donchenko, A. Gonoskov, E. Efimenko, A. Malyshev, I. Meyerov, and I. Surmin, Particle-in-Cell Plasma Simulation on Heterogeneous Cluster Systems, J. Comput. Sci. 3, 474 (2012).

[37] I. A. Surmin, S. I. Bastrakov, E. S. Efimenko, A. A. Gonoskov, A. V. Korzhimanov, and I. B. Meyerov, Particle-in-Cell laser-plasma simulation on Xeon Phi coprocessors, Comput. Phys. Commun. 202, 204 (2016).

[38] C. N. Harvey, A. Gonoskov, A. Ilderton, and M. Marklund, Quantum Quenching of Radiation Losses in Short Laser Pulses, Phys. Rev. Lett. 118, 105004 (2017).

[39] A. Gonoskov, I. Gonoskov, C. Harvey, A. Ilderton, A. Kim, M. Marklund, G. Mourou, and A. M. Sergeev, Probing Nonperturbative QED with Optimally Focused Laser Pulses, Phys. Rev. Lett. 111, 060404 (2013).

[40] H. R. Weller, M. W. Ahmeda, H. Gaoa, W. Tornowa, Y. K. Wua, M. Gai, and R. Miskimene, Research Opportunities at the Upgraded $\mathrm{HI} \gamma \mathrm{S}$ Facility, Prog. Part. Nucl. Phys. 62, 257 (2009).

[41] Spring-8, http://www.spring8.or.jp/en/.

[42] A2 Collaboration at the Mainz Microtron, http://www.kph .uni-mainz.de/eng/133.php. 